\title{
Nonsingular Terminal Sliding Mode Control of Uncertain Chaotic Gyroscope System Based on Disturbance Observer
}

\author{
Xin Ma, Yeguo Sun $\mathbb{D}^{D}$, and Fang Zhu \\ Department of Finance and Mathematics, Huainan Normal University, Huainan 232038, China \\ Correspondence should be addressed to Yeguo Sun; yeguosun@126.com
}

Received 3 November 2020; Revised 23 November 2020; Accepted 20 December 2020; Published 4 January 2021

Academic Editor: Ahmed Mostafa Khalil

Copyright $\odot 2021$ Xin Ma et al. This is an open access article distributed under the Creative Commons Attribution License, which permits unrestricted use, distribution, and reproduction in any medium, provided the original work is properly cited.

Based on disturbance observer, this paper develops a nonsingular terminal sliding mode control method for uncertain chaotic gyroscope system. Firstly, fuzzy logic system (FLS) is used to estimate the unknown function; then disturbance observer (DOB) is constructed to estimate the mixed disturbance, which consists of the fuzzy estimation error, external disturbance, and dead-zone input error. Subsequently, by using a nonsingular terminal sliding mode function, the control method proposed in this paper can achieve the sliding mode variable approaching a small neighborhood of zero and reduce chattering phenomenon of the tracking error and controller. Finally, comparative simulation results confirm the effectiveness of the method proposed in this paper.

\section{Introduction}

As an important sensor in navigation system, gyroscope was first used in ship navigation. With the development of science and technology, up to now, it has been widely used in aviation, aerospace, missile, automobile, and other related fields [1-4] requiring orientation and balance. However, the gyro system often exhibits chaotic phenomenon, which will damage its applications. In recent years, more and more attention has been paid to the control of the chaotic behavior of the gyroscope, for example, OGY control [5], linear feedback control [6], adaptive control [7, 8], and sliding mode control [9-22]. Among these methods, sliding mode control (SMC) is widely used because of its simple structure, fast response, and strong robustness to disturbance and unmodeled dynamics. However, due to the demand for new industrial applications and technological progress, some problems related to SMC are still the current research directions, such as disturbance elimination, selection of sliding mode surface, and integration with other control methods. For the gyro system, Moghani et al. [14] added fuzzy control based on the research of [13], using fuzzy inference engine to eliminate the discontinuity of the sign function of the SMC system at the arrival stage, which improved the performance of the system. Fazlyab et al. [15] studied a hybrid intelligent controller for vibratory gyroscopes in single-axis MEMS. An additional interval type-2 fuzzy SMC is used to minimize the effect of noise. Fang et al. [17] derived an $H_{\infty}$ control strategy based on Lyapunov function to achieve ideal attenuation of various external disturbances in MEMS gyroscope. Aiming at the trajectory tracking problem of an under-driven two-degrees-of-freedom control moment gyroscope, in order to increase the robustness, a controller, which was based on an adaptive neural network to compensate for unknown dynamics, was designed in [19]. Then, for this type of system, in addition to using the adaptive neural network algorithm in [19], Montoya-Chirez et al. [20] also proposed an adaptive model regressor scheme. Zhang et al. [21] studied the SMC with compound learning of the MEMS gyroscope, gave a series-parallel estimation model, and constructed the filter error to design the weights updating law of neural networks. By using prescribed performance control method, Xiang et al. [22] achieved the synchronization of two uncertain gyro systems.

However, although the control objective can be guaranteed by aforementioned control methods, the system uncertainties cannot be estimated accurately. In this paper, a novel control method is proposed to overcome this problem. The disturbance observation and fuzzy estimation parameters are integrated into the nonsingular terminal SMC 
(NTSMC), so that the controlled system can achieve finite time stability quickly and effectively. The main contributions of this paper are as follows: (1) The proposed control method can quickly stabilize the tracking error. (2) The mixed disturbance can be accurately estimated by proposed FLSs and DOB. (3) The proposed control method can avoid singular problem and chattering phenomena can also be reduced.

The organizational structure of this paper is as follows. In Section 2, the problem of a class of chaotic gyroscope systems is presented. The design and stability analysis of the control method are investigated in Section 3. In Section 4, comparative simulation results show the superiority of the proposed method. A short conclusion is given in Section 5.

\section{System Descriptions and Problem Formulations}

In this paper, a symmetrical gyroscope system with linear damping installed on a vibrating base is considered. The motion equation of the gyroscope system is given by the angle $\xi$ as

$$
\ddot{\xi}+A^{2} \frac{(1-\cos \xi)^{2}}{\sin ^{3} \xi}-B \sin \xi+C \dot{\xi}+D \dot{\xi}^{3}=F \sin \omega t \sin \xi,
$$

where $F \sin \omega t$ represents a parametric excitation, $C \dot{\xi}$ is linear damping term, $D \dot{\xi}^{3}$ is nonlinear damping term, and $A^{2}\left((1-\cos \xi)^{2} / \sin ^{3} \xi\right)-B \sin \xi$ is a nonlinear resilience force. Choosing parameters as $A^{2}=100, B=1, C=0.5, D=$ $0.05, F=35.7, \omega=2$ and states initial values of $\xi(0)=-1, \dot{\xi}$ $(0)=1$, the gyroscope system display chaotic behavior can be seen in Figure 1.

Define $x_{1}=\xi, x_{2}=\dot{\xi}$, and $x=\left[x_{1}, x_{2}\right]^{T}$; then the controlled gyroscope system (1) can be described as

$$
\left\{\begin{array}{l}
\dot{x}_{1}=x_{2} \\
\dot{x}_{2}=f(t, x)+d(t)+\chi(u(t))
\end{array}\right.
$$

where $f(t, x)=-A^{2}\left[\left(1-\cos \left(x_{1}\right)\right)^{2} / \sin ^{3}\left(x_{1}\right)\right]+B \sin \left(x_{1}\right)-$ $C x_{2}-D x_{2}^{3}+F \sin (\omega t) \sin \left(x_{1}\right), d(t)$ is an external disturbance, and $\chi(u(t))$ is the control input, which is affected by the dead zone. Similar to literature [22], $\chi(u(t))=\beta u$ $(t)+\sigma(u(t))$, and

$$
\sigma(u(t))=\left\{\begin{array}{l}
-\beta a_{1}, \quad \text { if } u(t)<a_{1}, \\
-\beta u(t), \quad \text { if } a_{1} \leq u(t) \leq a_{2}, \\
-\beta a_{2}, \quad \text { if } u(t)>a_{2},
\end{array}\right.
$$

where $\beta, a_{1}, a_{2}$ are design parameters. Clearly, $\sigma(u(t))$ is bounded. Meanwhile, we give the following assumptions.

Assumption 1. The nonlinear function $f(t, x)$ is unknown and bounded.

Assumption 2. The external disturbance $d(t)$ and its derivative are unknown and bounded.

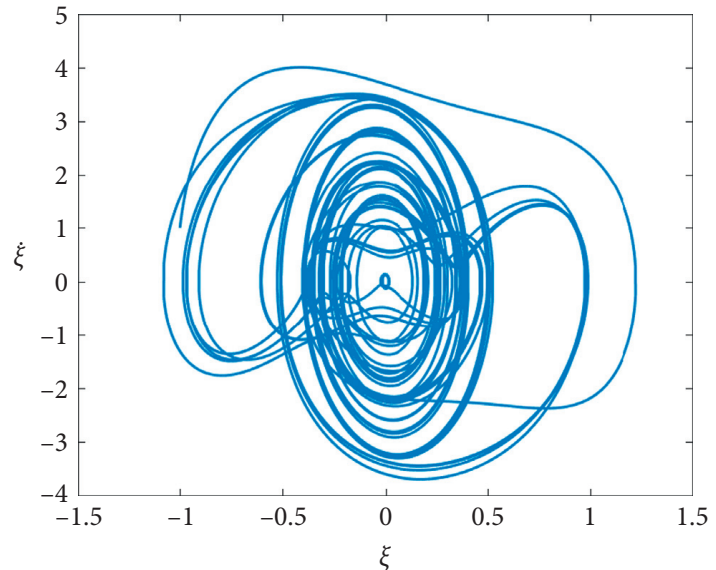

Figure 1: Phase plane trajectory of gyroscope system.

In this paper, the unknown function $f(t, x)$ is estimated by using the fuzzy logic system; for the relevant principles, please see $[23,24]$. The goal of this paper is to design a nonsingular fuzzy terminal sliding mode control method so that $x_{1}$ can track a reference signal $x_{d}$, where the reference signal $x_{d}$ and its second derivative $\ddot{x}_{d}$ are continuous and bounded.

\section{Control Design and Stability Analysis}

Let $e_{1}=x_{1}-x_{d}$; one has

$$
\left\{\begin{array}{l}
\dot{e}_{1}=e_{2}, \\
\dot{e}_{2}=\dot{x}_{2}-x_{d}=f(t, x)-x_{d}+d(t)+\beta u(t)+\sigma(u(t)),
\end{array}\right.
$$

and define $F(t, x)=\lambda f(t, x)$, by using fuzzy logic system, which can be expressed as

$$
\left\{F(t, x)=\eta^{* T} \varpi_{F}(x)+\rho(x),\right.
$$

where $\lambda>0$ is design parameter, $\eta^{*}$ is the optimal approximation vector, $\Phi_{F}(x)$ is the basis function vector, $\rho(x)$ is a fuzzy estimation error, and, according to [23], $\rho(x)$ is bounded.

From the relation between $F(t, x)$ and $f(t, x)$, one gets

$$
\begin{aligned}
f(t, x) & =\lambda^{-1} F(t, x)=\lambda^{-1}\left[\eta^{* T} \omega_{F}(x)+\rho(x)\right], \\
& =\lambda^{-1} \widetilde{\eta}^{T} \omega_{F}(x)+\lambda^{-1} \widehat{\eta}^{T} \omega_{F}(x)+\lambda^{-1} \rho(x),
\end{aligned}
$$

and let $\widetilde{\eta}=\eta^{*}-\hat{\eta}$, where $\hat{\eta}$ is an estimate of $\eta^{*}$. Then equation (4) can be written as

$$
\left\{\begin{array}{l}
\dot{e}_{1}=e_{2}, \\
\dot{e}_{2}=\lambda^{-1} \widetilde{\eta}^{T} \omega_{F}(x)+\lambda^{-1} \widehat{\eta}^{T} \omega_{F}(x)-\ddot{x}_{d}+\bar{d}(t)+\beta u(t),
\end{array}\right.
$$

where $\bar{d}(t)=\lambda^{-1} \rho(x)+d(t)+\sigma(u(t))$ (we denote $\bar{d}(t)$ as mixed disturbance). It is easy to know that $|\bar{d}(t)|$ is bounded; that is, $|\bar{d}(t)| \leq d^{\max }, d^{\max }$ is an unknown positive constant.

Define the following terminal sliding mode as 


$$
s=e_{2}+\varsigma e_{1}+e_{1}^{\tau}
$$

where $0<\tau=q / p<1, q$ and $p$ are positive odd constants, and $\varsigma$ is positive constant.

Remark 1. Compared with the traditional sliding mode in [12], the terminal sliding mode in (8) can achieve a fast approach speed for $e_{1}$; and, to avoid the singularity problem, we define $\tau e_{1}^{\tau-1} e_{2}$ as follows:

$$
\tau e_{1}^{\tau-1} e_{2}= \begin{cases}\tau e_{1}^{\tau-1} e_{2}, & \text { for } e_{1} \neq 0, e_{2} \neq 0, \\ \tau \varepsilon_{1}^{\tau-1} e_{2}, & \text { for } e_{1}=0, e_{2} \neq 0, \\ 0, & \text { for } e_{1}=0, e_{2}=0,\end{cases}
$$

where $\epsilon_{1}$ is a small positive number.Taking derivative of $s$, one has

$$
\begin{aligned}
\dot{s}= & \dot{e}_{2}+\varsigma \dot{e}_{1}+\tau e_{1}^{\tau-1} \dot{e}_{1}, \\
= & \lambda^{-1} \widetilde{\eta}^{T} \varpi_{F}(x)+\lambda^{-1} \widehat{\eta}^{T} \varpi_{F}(x)-\ddot{x}_{d}+\bar{d}(t) \\
& +\beta u(t)+\varsigma e_{2}+\tau e_{1}^{\tau-1} e_{2} .
\end{aligned}
$$

According to (10), the control method is designed as

$$
u=u_{1}+u_{2}+u_{3}
$$

with

$$
\begin{aligned}
& u_{1}=\frac{1}{\beta}\left[\ddot{x}_{d}-\varsigma e_{2}-\tau e_{1}^{\tau-1} e_{2}\right], \\
& u_{2}=\frac{1}{\beta}\left[-\widehat{\bar{d}}(t)-\lambda^{-1} \hat{\eta}^{T} \varpi_{F}(x)\right], \\
& u_{3}=\frac{1}{\beta}\left[-l_{1} s-l_{2}|s|^{a} \operatorname{sign}(s)\right],
\end{aligned}
$$

where $\widehat{\bar{d}}$ is the estimate of $\bar{d}, l_{1}$ and $l_{2}$ are positive design parameters, and $0<a<1$.

Let $\bar{d}=\bar{d}-\widehat{\bar{d}}$. From equations (10)-(12), one gets

$$
\dot{s}=-l_{1} s-l_{2}|s|^{a} \operatorname{sign}(s)+\widetilde{\bar{d}}+\lambda^{-1} \tilde{\eta}^{T} \omega_{F}(x) .
$$

For fuzzy estimation parameter $\tilde{\eta}$, the adaptive law is given as

$$
\dot{\hat{\eta}}=k_{1}\left(s \lambda^{-1} ळ_{F}(x)-k_{2} \hat{\eta}\right)
$$

where $k_{1}$ and $k_{2}$ are positive design parameters.

Then, we construct the following disturbance observer:

$$
\left\{\begin{array}{l}
\widehat{\bar{d}}=m\left(e_{2}-z\right), \\
\dot{z}=\lambda^{-1} \widehat{\eta}^{T} \omega_{F}(x)+\beta u(t)+\widehat{\bar{d}}-x_{d}-m^{-1} s,
\end{array}\right.
$$

where $m$ is a positive design parameter.

From the disturbance observation error $\widetilde{\bar{d}}=\bar{d}-\widehat{\bar{d}}$ and equation (15), we can get

$$
\begin{aligned}
\dot{\overline{\bar{d}}} & =\dot{\bar{d}}-\dot{\overline{\bar{d}}}=\dot{\bar{d}}-m\left(\dot{e}_{2}-\dot{z}\right), \\
& =\dot{\bar{d}}-m\left(\widetilde{\bar{d}}+t \lambda^{-1} n \tilde{\eta}^{T} q \Phi_{F} h(x)_{+} x m^{-1} 7 s\right) .
\end{aligned}
$$

From the above analysis, we obtain the following theorem.

Theorem 1. Consider the uncertain chaotic gyroscope system (1), which satisfies Assumptions 1 and 2; the parameter adaptation law (14), disturbance observer (15), and terminal sliding mode controller (11) can guarantee that all of closedloop signals $s, \widetilde{\eta}, \bar{d}$ are bounded, and the sliding mode variable $s$ converges to small neighborhood of zero in finite time.

Proof. Consider the Lyapunov function candidate as

$$
V=\frac{1}{2}\left[s^{2}+\widetilde{\bar{d}}^{2}+\frac{1}{k_{1}} \tilde{\eta}^{T} \widetilde{\eta}\right] .
$$

We have

$$
\begin{aligned}
s \dot{s} & =s\left[-l_{1} s-l_{2}|s|^{a} \operatorname{sign}(s)+\widetilde{\bar{d}}+\lambda^{-1} \widetilde{\eta}^{T} \varpi_{F}(x)\right], \\
& =-l_{1} s^{2}-l_{2}|s|^{a+1}+s \widetilde{\bar{d}}+s \lambda^{-1} \widetilde{\eta}^{T} \varpi_{F}(x),
\end{aligned}
$$

$\widetilde{\overline{\bar{d}}}=\widetilde{\bar{d}}-\widetilde{\bar{d}} m \lambda^{-1} \widetilde{\eta}^{T} \omega_{F}(x)-m \widetilde{\bar{d}}^{2}-\widetilde{\bar{d}} s$,

$$
\begin{aligned}
\frac{1}{k_{1}} \widetilde{\eta}^{T} \dot{\tilde{\eta}} & =\frac{1}{k_{1}} \widetilde{\eta}^{T}(-\dot{\hat{\eta}})=-\frac{1}{k_{1}} \widetilde{\eta}^{T}\left[k_{1}\left(s \lambda^{-1}{\varpi_{F}}(x)\right)-k_{2} \hat{\eta}\right], \\
& =-\lambda^{-1} s \widetilde{\eta}^{T} \varpi_{F}(x)+k_{2} \widetilde{\eta}^{T} \widehat{\eta} .
\end{aligned}
$$

Therefore, $\dot{V}$ can be expressed as

$$
\begin{aligned}
& \dot{V}=s \dot{\mathcal{s}}+\frac{\widetilde{\tilde{d}}}{k_{1}}+\frac{1}{\tilde{\eta}^{T}} \dot{\tilde{\eta}} \\
& =-l_{1} s^{2}-l_{2}|s|^{a+1}+s \tilde{\bar{d}}+s \lambda^{-1} \widetilde{\eta}^{T} \varpi_{F}(x)+\tilde{\bar{d}} \\
& -m \lambda^{-1} \widetilde{\bar{d}} \widetilde{\eta}^{T} \omega_{F}(x) \\
& -m \widetilde{\bar{d}}^{2}-\widetilde{\bar{d}} s-\lambda^{-1} s \widetilde{\eta}^{T} \omega_{F}(x)+k_{2} \widetilde{\eta}^{T} \widehat{\eta} \\
& =-l_{1} s^{2}-l_{2}|s|^{a+1}+\tilde{\bar{d} \dot{d}}-m \lambda^{-1} \widetilde{\bar{d}} \widetilde{\eta}^{T} \Phi_{F}(x) \\
& -m \widetilde{\bar{d}}^{2}+k_{2} \widetilde{\eta}^{T} \widehat{\eta} .
\end{aligned}
$$

The following inequalities hold:

$$
\begin{aligned}
\frac{\widetilde{\bar{d}}}{\mathrm{~d}} & \leq \frac{1 \widetilde{\bar{d}}^{2}}{2}+\frac{1 \dot{\bar{d}}^{2},}{2} \\
-m \lambda^{-1} \widetilde{\bar{d}} \widetilde{\eta}^{T} \omega_{F}(x) & \leq m \lambda^{-1}\left(\frac{1}{2} \widetilde{\eta}^{T} \widetilde{\eta}+\frac{1}{2} \Upsilon^{2} \widetilde{\bar{d}}^{2}\right), \\
k_{2} \widetilde{\eta}^{T} \widehat{\eta} & =k_{2} \widetilde{\eta}^{T}\left(\eta^{*}-\widetilde{\eta}\right) \leq-\frac{k_{2}}{2} \widetilde{\eta}^{T} \widetilde{\eta}+\frac{k_{2}}{2} \eta^{* T} \eta^{*},
\end{aligned}
$$


where $\left\|\varpi_{F}(x)\right\| \leq \Upsilon$.

Therefore, substituting (20) into (19), one obtains

$$
\begin{aligned}
& \dot{V} \leq-l_{1} s^{2}-l_{2}|s|^{a+1}+\frac{1}{2} \widetilde{d}^{2}+\frac{1}{2} \dot{\bar{d}}^{2}-m \tilde{\bar{d}}^{2} \\
& +m \lambda^{-1}\left(\frac{1}{2} \widetilde{\eta}^{T} \widetilde{\eta}+\frac{1}{2} \Upsilon^{2} \widetilde{\bar{d}}^{2}\right) \\
& -\frac{k_{2}}{2} \widetilde{\eta}^{T} \widetilde{\eta}+\frac{k_{2}}{2} \eta^{* T} \eta^{*}=-l_{1} s^{2}-l_{2}|s|^{a+1}-k_{d} \widetilde{\bar{d}}^{2}-k_{\eta}\|\widetilde{\eta}\|^{2}+p_{0},
\end{aligned}
$$

where $k_{d}=m-.(2 / 2)-(1 / 2) m \lambda^{-1} \Upsilon^{2}, k_{\eta}=\left(k_{2} / 2\right)-(1 / 2)$ $m \lambda^{-1}, p_{0}=(1 / 2) \bar{d}+\left(k_{2} / 2\right) \eta^{* T} \eta^{*}$.

By selecting parameters $m, \lambda$, and $k_{2}$ such that $k_{d}>0, k_{\eta}>0$, one has

$$
\dot{V} \leq-c_{1} V+p_{0},
$$

where $c_{1}=\min \left\{2 l_{1}, 2 k_{d}, 2 k_{\eta}\right\}$.

From (22), one gets

$$
0 \leq V \leq \frac{p_{0}}{c_{1}}+\left[V(0)-\frac{p_{0}}{c_{1}}\right] e^{-c_{1} t} .
$$

It is known that $V \longrightarrow\left(p_{0} / c_{1}\right)$, as $t \longrightarrow \infty$. Then all the signals involved in (17) are bounded. Let $\theta=\widetilde{\bar{d}}+\lambda^{-1} \widetilde{\eta}^{T} \varpi_{F}$ $(x)$.

Then (13) can be written as

$$
\dot{s}=-l_{1} s-l_{2}|s|^{a} \operatorname{sign}(s)+\theta .
$$

Because $\tilde{\bar{d}}$ and $\widetilde{\eta}$ are bounded, there exists an unknown constant $R$ such that $|\theta| \leq R$.

If $s$ is not zero, rewrite (24) as

$$
\dot{s}=-\left(l_{1}-\frac{\theta}{s}\right) s-l_{2}|s|^{a} \operatorname{sign}(s) .
$$

Let $l_{1}=(R /|s|)+r_{1}$, and let $\widetilde{V}=(1 / 2) s^{2}$; then we have

$$
\dot{\tilde{V}}=s \dot{s} \leq-r_{1} s^{2}-l_{2}|s|^{a+1}=-2 r_{1} \tilde{V}-2 l_{2} \widetilde{V}^{(a+1 / 2)} \text {. }
$$

As we all know, $s$ will converge to the regions $\Omega=\left\{|s|:|s| \leq\left(R / l_{1}-r_{1}\right)\right\}$ in finite time.

Remark 2. Reference [12] used sliding mode control method to achieve the synchronization of a class of chaotic systems. However, in [12], just assuming that the disturbance is bounded and through the sign function to eliminate the influence of the external disturbance, the controller can make the state enter the sliding mode surface which is designed, but it will produce a large jitter phenomenon. Moreover, the method cannot understand the influence of the external disturbance and unknown function on the system, so the disturbance observer is designed in this paper. On the one hand, it targets accurately estimating the mixed disturbance, which is composed of the dead-zone input error, fuzzy estimation error, and the external disturbance. On the other hand, compared with the traditional control

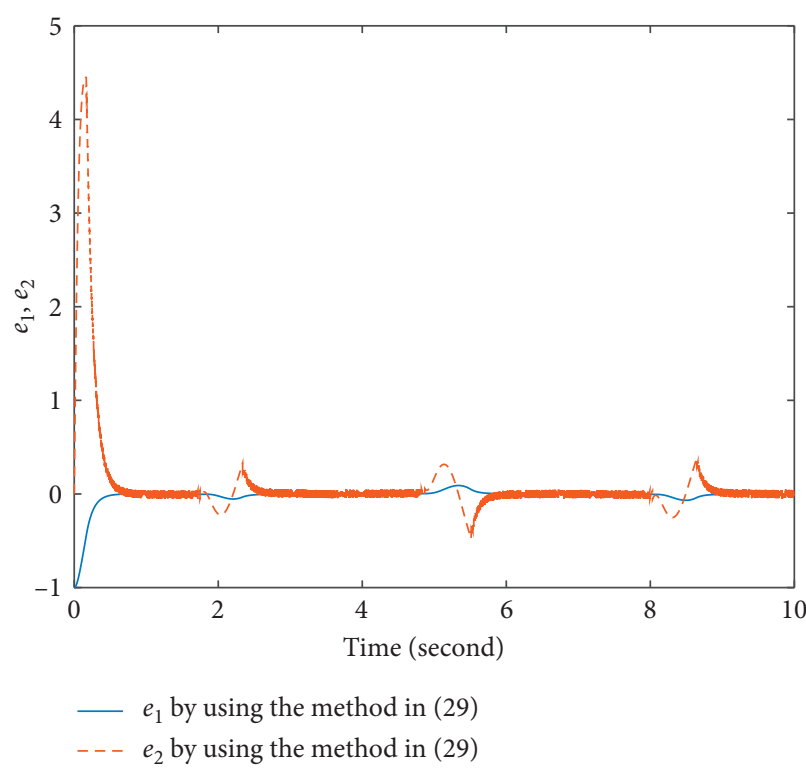

Figure 2: Trajectories of $e_{1}$ and $e_{2}$ by using the method in (27).

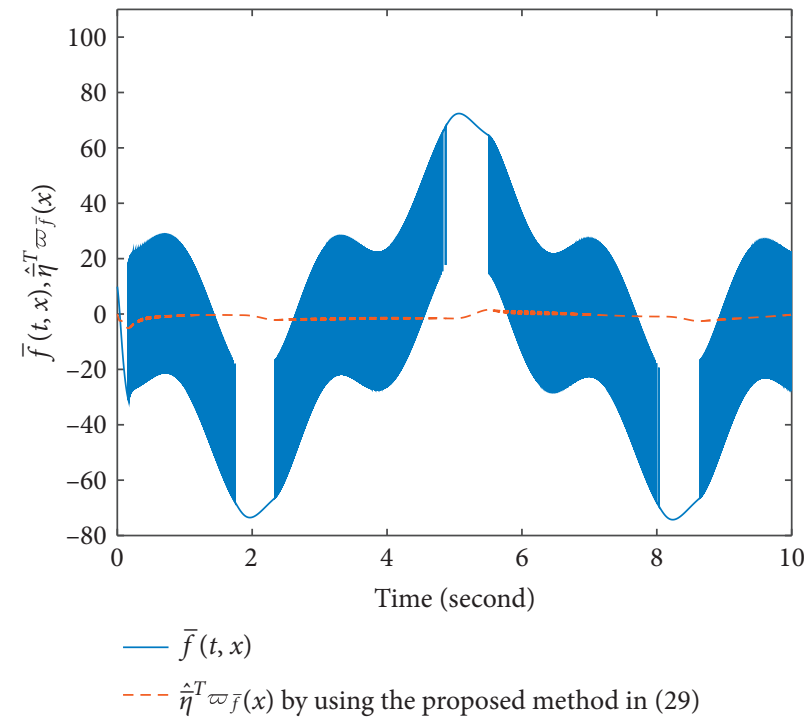

FIgURE 3: Trajectories of $\bar{f}(t, x)$ and $\widehat{\bar{\eta}}^{T} \Phi_{\bar{f}}(x)$ by using the method in (27).

method, the control effect will be further improved and the jitter phenomenon will be reduced. Therefore, this paper can be regarded as a further study of [12].

\section{Numerical Simulations}

In this section, the parameters of the chaotic system are selected as $A^{2}=100, B=1, C=0.5, D=0.05, F=35.7, \omega=$ 2, with initial values of $\xi(0)=-1, \dot{\xi}(0)=1$ and reference signal $x_{d}=\sin (t)$. For comparison with the proposed method in (11), the traditional control method combining linear sliding mode and fuzzy logic system is designed as follows: 


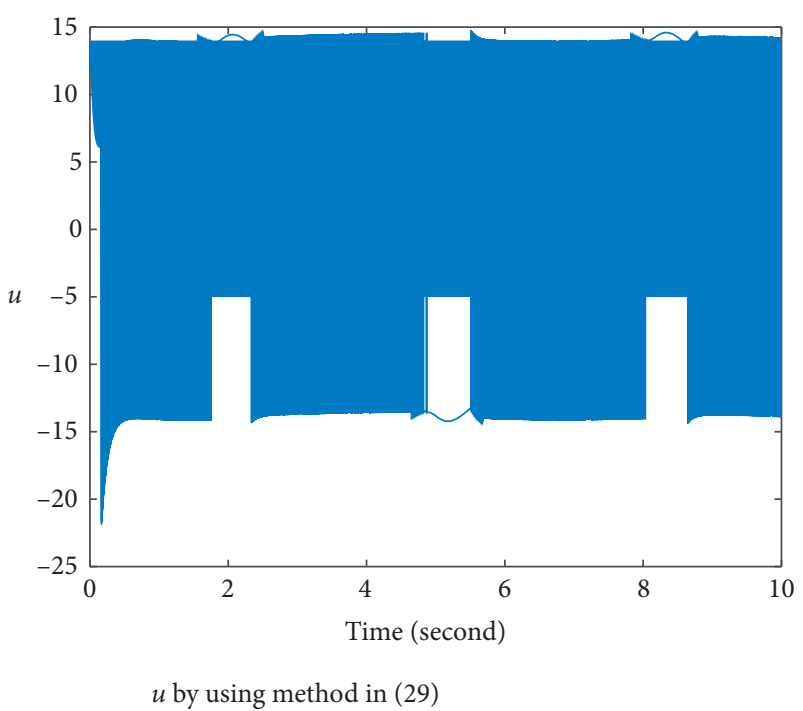

Figure 4: Trajectory of $u$ by using the method in (27).

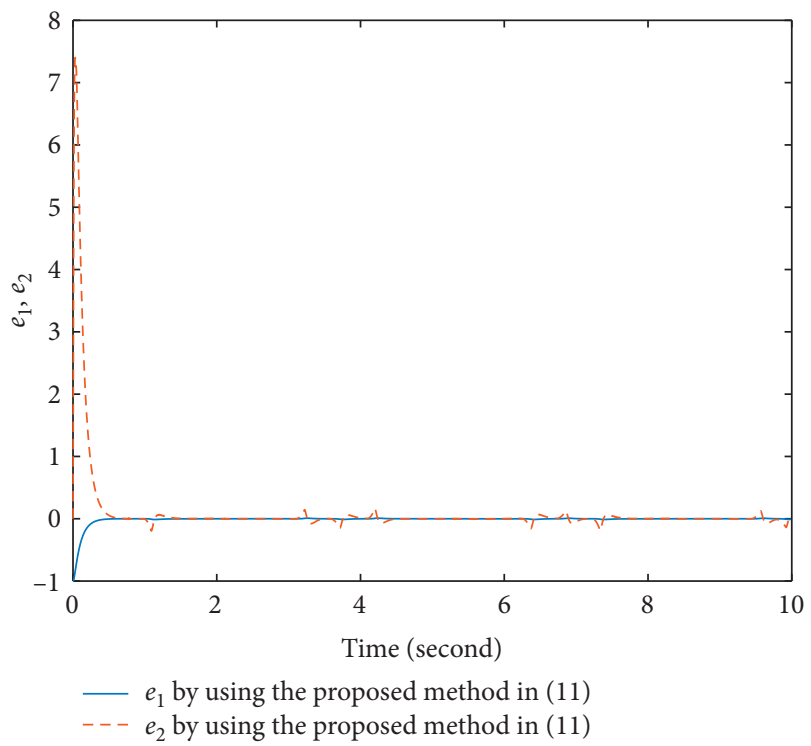

Figure 5: Trajectories of $e_{1}$ and $e_{2}$ by using the proposed method in (11).

$$
\begin{aligned}
& \bar{s}=e_{2}+\varsigma e_{1}, \\
& u=\frac{1}{\beta}\left[-\widehat{\bar{\eta}}^{T} \Phi_{\bar{f}}(x)+\ddot{x}_{d}-\varsigma e_{2}-\bar{\gamma} \operatorname{sign}(\bar{s})\right], \\
& \dot{\bar{\eta}}=k_{1}\left(\bar{s} \Phi_{\bar{f}} t(x) n-q k_{2} h \widehat{\bar{\eta}}\right),
\end{aligned}
$$

where $\hat{\bar{\eta}}^{T} \Phi_{\bar{f}}(x)$ is the fuzzy estimation of $f(t, x)+d(t)+\sigma(u(t))$, and $d(t)=\cos ((3 / 5) t)$. Define five Gaussian membership functions centered at $-5,-2.5,0,2.5$, 5 with a variance equal to 1.2 . The initial value of $\widehat{\bar{\eta}}(0)=0$, and other parameters are selected as $\bar{\gamma}=70, k_{1}=30, k_{2}=$ $0.5, \varsigma=10, \beta=a_{1}=a_{2}=5$. By using the method in (27), the corresponding simulation results are shown in Figures 2-4.

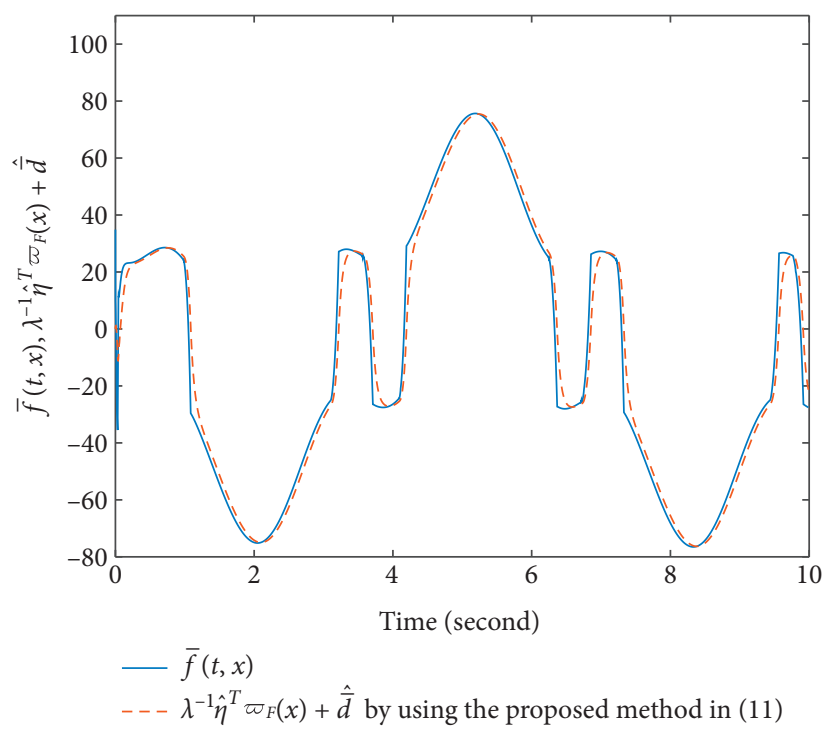

Figure 6: Trajectories of $\bar{f}(t, x)$ and $\lambda^{-1} \widehat{\eta}^{T} \oplus_{F}(x)+\widehat{\bar{d}}(t)$ by using the proposed method in (11).

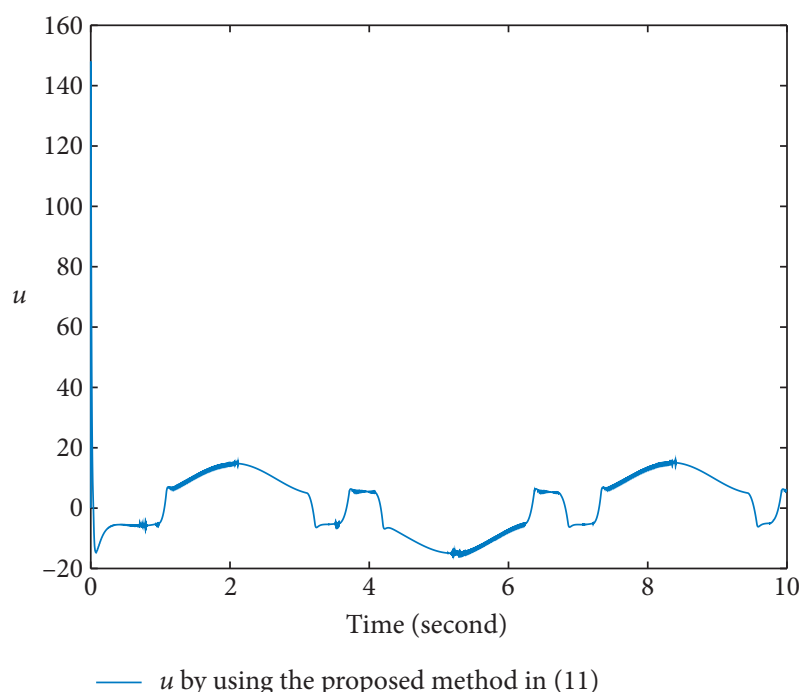

FIGURE 7: Trajectory of $u$ by using the proposed method in (11).

It can be seen from Figure 2 that the tracking error $e_{1}$ and its derivative $e_{2}$ approach the neighborhood of zero, but the chattering phenomenon of $e_{2}$ is obvious. Figure 3 exhibits that the fuzzy estimation $\hat{\bar{\eta}}^{T^{2}} \Phi_{\bar{f}}(x)$ cannot effectively estimate the unknown function $\bar{f}(t, x)$ and Figure 4 shows that the controller $u$ has chattering phenomenon. Under the same parameters and initial values of the method in (27), the other parameters and initial values are selected as $m=20, \lambda=5, \tau=(3 / 5)$ and $\epsilon_{1}=0.05, z(0)=0$ for the proposed method in (11), and the simulation results of the proposed method in (11) are shown in Figures 5-7.

Obviously, compared with Figure 2, the control effect of $e_{1}$ and its derivative $e_{2}$ is improved in Figure 5, and the chattering phenomenon is also reduced. From Figure 6, we can find that the estimation effect of the proposed method in (11) for $\bar{f}(t, x)$ is more better than that of the method in 
(27), and the chattering phenomenon of controller $u$ is also reduced in Figure 7. The above simulation results confirm that the method proposed in (11) in this paper is more effective.

\section{Conclusion}

In this paper, a terminal sliding mode control method is proposed for uncertain chaotic gyroscope system; based on disturbance observer and FLSs, the mixed disturbance can be estimated accurately. By using a nonsingular terminal sliding mode function, the proposed control method can achieve the sliding mode variable approaching a small neighborhood of zero. Then, the boundedness of all closedloop signals is proved according to Lyapunov theory. Compared with the traditional sliding mode control method, simulation results verify that the method proposed in this paper has better control performance.

\section{Data Availability}

All datasets generated for this study are included in the manuscript.

\section{Conflicts of Interest}

The authors declare that there are no conflicts of interest regarding the publication of this paper.

\section{Acknowledgments}

The authors gratefully acknowledge the support of the Natural Science Foundation of Anhui Province project (2008085MF200) and the Natural Science Research Projects in Anhui Universities (KJ2019ZD48 and KJ2019A0695).

\section{References}

[1] T. Yuhara, T. Kumagai, H. Soekawa, and H. Kajioka, "Fiberoptic gyroscopes for automotive applications," Journal of Circuits, Systems and Computers, vol. 5, no. 1, pp. 17-36, 1995.

[2] I. Jikuya, K. Fujii, and K. Yamada, "Attitude maneuver of spacecraft with a variable-speed double-gimbal control moment gyro," Advances in Space Research, vol. 58, no. 7, pp. 1303-1317, 2016.

[3] A. Donaire and T. Perez, "Energy-based nonlinear control of ship roll gyro-stabiliser with precession angle constraints," IFAC Proceedings Volumes, vol. 46, no. 33, pp. 328-333, 2013.

[4] Z. Koruba, I. Krzysztofik, and Z. Dziopa, "An analysis of the gyroscope dynamics of an anti-aircraft missile launched from a mobile platform," Bulletin of the Polish Academy of Sciences: Technical Sciences, vol. 58, no. 4, pp. 651-656, 2010.

[5] S. M. Abtahi and S. H. Sadati, "Chaos control in attitude dynamics of a gyrostat satellite based on linearised Poincare' map estimation by support vector machine," Proceedings of the Institution of Mechanical Engineers, Part K: Journal of Multi-Body Dynamics, vol. 227, no. 3, pp. 302-312, 2013.

[6] M. Sadeghpour, M. Khodabakhsh, and H. Salarieh, "Intelligent control of chaos using linear feedback controller and neural network identifier," Communications in Nonlinear
Science and Numerical Simulation, vol. 17, no. 12, pp. 47314739, 2012.

[7] M. M. Zirkohi and S. khorashadizadeh, "Chaos synchronization using higher-order adaptive pid controller," $A E U$ -International Journal of Electronics and Communications, vol. 94, pp. 157-167, 2018.

[8] H. Liu, Y. Pan, and J. Cao, "Composite learning adaptive dynamic surface control of fractional-order nonlinear systems," IEEE Transactions on Cybernetics, vol. 50, no. 6, pp. 2557-2567, 2020.

[9] S. Etemadi, A. Alasty, and H. Salarieh, "Synchronization of chaotic systems with parameter uncertainties via variable structure control," Physics Letters A, vol. 357, no. 1, pp. 17-21, 2006.

[10] Y. Zhou, H. Liu, J. Cao, and S. Li, "Composite learning fuzzy synchronization for incommensurate fractional-order chaotic systems with time-varying delays," International Journal of Adaptive Control and Signal Processing, vol. 33, no. 12, pp. 1739-1758, 2019.

[11] S. Ha, H. Liu, S. Li, and A. Liu, "Backstepping-based adaptive fuzzy synchronization control for a class of fractional-order chaotic systems with input saturation," International Journal of Fuzzy Systems, vol. 21, no. 5, pp. 1571-1584, 2019.

[12] M. Roopaei, M. Zolghadri Jahromi, R. John, and T.-C. Lin, "Unknown nonlinear chaotic gyros synchronization using adaptive fuzzy sliding mode control with unknown dead-zone input," Communications in Nonlinear Science and Numerical Simulation, vol. 15, no. 9, pp. 2536-2545, 2010.

[13] J. Fei and C. Batur, "A novel adaptive sliding mode control with application to mems gyroscope," ISA Transactions, vol. 48, no. 1, pp. 73-78, 2009.

[14] M. R. Moghanni, J. Keighobadi, and A. Ghanbari, "Fuzzy adaptive sliding mode controller for mems vibratory rate gyroscope," IFAC Proceedings Volumes, vol. 44, no. 1, pp. 4192-4197, 2011.

[15] M. Fazlyab, M. Z. Pedram, H. Salarieh, and A. Alasty, "Parameter estimation and interval type-2 fuzzy sliding mode control of a $\mathrm{z}$-axis mems gyroscope," ISA Transactions, vol. 52, no. 6, pp. 900-911, 2013.

[16] F. Min and A. C. J. Luo, "On the projective function synchronization of chaos for two gyroscope systems under sinusoidal constraints," International Journal of Dynamics and Control, vol. 1, no. 3, pp. 203-213, 2013.

[17] Y. Fang, J. Fei, and S. Wang, "H-infinity control of MEMS gyroscope using T-S fuzzy model," IFAC-PapersOnLine, vol. 48, no. 14, pp. 241-246, 2015.

[18] M. Rahmani, "Mems gyroscope control using a novel compound robust control," ISA Transactions, vol. 72, pp. 37-43, 2018.

[19] Y. Jia and A. K. Misra, "Robust trajectory tracking control of a dual-arm space robot actuated by control moment gyroscopes," Acta Astronautica, vol. 137, pp. 287-301, 2017.

[20] J. Montoya-Cháirez, V. Santibáñez, and J. Moreno-Valenzuela, "Adaptive control schemes applied to a control moment gyroscope of 2 degrees of freedom," Mechatronics, vol. 57, pp. 73-85, 2019.

[21] R. Zhang, T. Shao, W. Zhao, A. Li, and B. Xu, "Sliding mode control of mems gyroscopes using composite learning," Neurocomputing, vol. 275, pp. 2555-2564, 2018.

[22] W. Xiang, Y. Sun, N. Li, and C. Yang, "Uncertain chaotic gyros synchronization using adaptive fuzzy prescribed performance control with unknown dead-zone input," International Journal of Innovative Computing Information and Control, vol. 13, no. 2, pp. 429-440, 2017. 
[23] A. Boulkroune, M. Tadjine, M. M'Saad, and M. Farza, "Fuzzy adaptive controller for mimo nonlinear systems with known and unknown control direction," Fuzzy Sets and Systems, vol. 161 , no. 6 , pp. $797-820,2010$.

[24] B. Xu, "Composite learning finite-time control with application to quadrotors," IEEE Transactions on Systems, Man, and Cybernetics: Systems, vol. 48, no. 10, pp. 1806-1815, 2018. 